\title{
Prognostic Factors for Risk Stratification of Patients with Recurrent or Metastatic Pancreatic Adenocarcinoma Who Were Treated with Gemcitabine-Based Chemotherapy
}

\author{
Inkeun Park, $\mathrm{MD}^{1}$ \\ Seung Joon Choi, MD, PhD² \\ Young Saing Kim, MD \\ Hee Kyung Ahn, MD' \\ Junshik Hong, MD \\ Sun Jin Sym, MD, PhD' \\ Jinny Park, MD, PhD ${ }^{1}$ \\ Eun Kyung Cho, MD, PhD' \\ Jae Hoon Lee, MD, $\mathrm{PhD}{ }^{1}$ \\ Yong Ju Shin, BSPH ${ }^{3}$ \\ Dong Bok Shin, MD, PhD'
}

\section{Purpose}

The aim of this study was to verify prognostic factors including sarcopenia in patients with recurrent or metastatic pancreatic cancer receiving gemcitabine-based chemotherapy.

\section{Materials and Methods}

Medical records and computed tomography scan of consecutive patients treated with palliative gemcitabine-based chemotherapy from 2008 to 2014 were reviewed. The lumbar skeletal muscle index at third lumbar spine level was computed, and together with clinicolaboratory factors, univariate and multivariable analyses for overall survival (OS) were performed.

\section{Results}

A total of 88 patients were found. Median age was 65 years, and male patients were predominant (67.0\%). Most patients had initially metastatic disease (72.7\%), and gemcitabine monotherapy was administered in 29 patients (33.0\%) while gemcitabine plus erlotinib was administered in 59 patients (67.0\%). Seventy-six patients (86.3\%) had sarcopenia. With a median follow-up period of 44.3 months (range, 0.6 to 44.3 months), median OS was 5.35 months ( $95 \%$ confidence interval [Cl], 4.11 to 6.59 ). In univariate and multivariable analysis, high carcinoembryonic antigen level (hazard ratio [HR], 4.18; $95 \% \mathrm{Cl}, 1.95$ to 8.97; $\mathrm{p}<0.001$ ), initially metastatic disease ( $\mathrm{HR}, 3.37 ; 95 \% \mathrm{Cl}, 1.55$ to $7.32 ; \mathrm{p}=0.002)$, sarcopenia (HR, 2.97; 95\% Cl, 1.20 to 7.36; $p=0.019$ ), neutrophilia (HR, 2.94; 95\% Cl, 1.27 to 6.79; $\mathrm{p}=0.012$ ), and high lactate dehydrogenase level (HR, 1.96; $95 \% \mathrm{Cl}, 1.07$ to 3.58 ; $p=0.029$ ) were identified as independent prognostic factors for OS.

\section{Conclusion}

Five independent prognostic factors in patients with recurrent or metastatic pancreatic cancer who received gemcitabine-based chemotherapy were identified. These findings may be helpful in prediction of prognosis in clinical practice and can be used as a stratification factor for clinical trials.
Division of Hematology and Oncology,

Department of Internal Medicine,

Gachon University Gil Medical Center,

21 Namdong-daero 774beon-gil, Namdong-gu, Incheon 21565, Korea

Tel: 82-32-460-8371

Fax: 82-32-460-2391

E-mail: dbs@gilhospital.com

Received July 13, 2015

Accepted March 14, 2016

Published Online March 23, 2016

*Inkeun Park and Seung Joon Choi contributed equally to this work.
Key words

Pancreatic neoplasms, Gemcitabine, Drug therapy, Sarcopenia, Prognosis

\section{Introduction}

Pancreatic adenocarcinoma is a leading cause of cancerrelated death worldwide, with approximately 330,400 deaths in 2012 [1]. The majority of patients with pancreatic cancer present with locally advanced or metastatic disease, and less than $20 \%$ of patients can proceed with curative intent surgery [2]. The prognosis of unresectable or metastatic disease is extremely poor, as the median overall survival (OS) of 
patients is only 6 to 12 months even in the clinical trial setting [3-6], and 5-year OS rate of all-stage pancreatic cancer is under 10\%, which did not change from 1993 until 2012 in Korea [7].

Gemcitabine became standard first line chemotherapy for metastatic or recurrent pancreatic cancer after survival benefit was confirmed compared to 5 -fluorouracil in a randomized phase III trial [3]. As of 2015, the efficacy of several agents in pancreatic cancer, including gemcitabine and erlotinib combination, gemcitabine and nanoalbumin paclitaxel combination, and a combination chemotherapy regimen consisting of oxaliplatin, irinotecan, fluorouracil, and leucovorin (FOLFIRINOX), has been demonstrated [4-6]. FOLFIRINOX prolonged median OS from 6.8 months with gemcitabine to 11.1 months [5]; however, considerable toxicities preclude their dissemination in real world practice. Because most patients present with systemic manifestations of the disease including asthenia, anorexia, and weight loss [2], gemcitabine-based chemotherapy is still the most widely used first-line chemotherapy regimen [8].

Cachexia, which is prevalent in pancreatic cancer even in the resectable stage [9], has been shown to cause worsened prognosis and has also been associated with impairment of physical function, increased psychological distress, and low quality of life $[9,10]$. Sarcopenia, involuntary loss of skeletal muscle mass and/or strength, has recently been acknowledged as the major component of cancer cachexia [11]. Association of sarcopenia and survival in advanced pancreatic cancer has been reported in several studies [12-14]; however, these studies are heterogeneous in definition of cachexia and sarcopenia, patient population, and treatment. In addition, in survival analyses, clinically relevant factors including metastatic organ, laboratory values, and performance status were missed in these articles.

Therefore, the aim of this study was to evaluate prognostic factors including sarcopenia in patients with recurrent or metastatic pancreatic adenocarcinoma treated with gemcitabine-based chemotherapy.

\section{Materials and Methods}

The medical records of consecutive patients diagnosed with pancreatic cancer at Gachon University Gil Medical Center, Incheon, Republic of Korea from March 2008 to March 2014 were screened for the study using a prospectively maintained pancreas-biliary cancer database. Among this population, the selection criteria for analysis were as follows: patients who had histologically or cytologically proven metastatic or recurrent pancreatic adenocarcinoma, adequate quality of abdominal computed tomography (CT) scan within 4 weeks of initiation of chemotherapy, and underwent systemic treatment consisting of gemcitabine or gemcitabine plus erlotinib. Patients with ampulla of Vater cancer, distal common bile duct cancer, or neuroendocrine carcinoma of the pancreas were excluded.

Clinicopathological data included age, sex, height, weight, recent weight loss (involuntary weight loss of more than 5\% over past 6 months), Eastern Cooperative Oncology Group performance status (ECOG PS), tumor histology, differentiation, baseline laboratory values (complete blood count with differential count and serum chemistry), serum tumor markers (carcinoembryonic antigen [CEA] and carbohydrate antigen 19-9 [CA19-9]), disease status (recurrent of metastatic), metastatic site, previous treatment history, chemotherapy regimen, chemotherapy response, survival status and date of administration of chemotherapy and the last follow-up. Response evaluation was performed retrospectively according to Response Evaluation Criteria in Solid Tumors ver. 1.1 using follow-up radiographic images obtained every 6-8 weeks during treatment. The study was approved by the Institutional Review Board of Gachon University Gil Medical Center.

\section{Measurement and definition of sarcopenia}

A radiologist (S.J.C) with extensive experience in abdominal imaging study identified a single axial image at the level of the third lumbar vertebrae (L3) on which both transverse processes were fully observed. The skeletal muscle at L3 level includes the rectus abdominus; internal, external, and lateral obliques; psoas; quadratus lumborum; and erector spinae muscles. The muscle boundaries were drawn manually using the area-measuring tool on the Picture Archiving Communication System (Infinitt PACS, Seoul, Korea). Cross-sectional areas $\left(\mathrm{cm}^{2}\right)$ were computed automatically by summing tissue pixels and multiplying by pixel surface area. Image analysis was performed by one investigator (S.J.C.) who was blinded to patient outcomes. The lumbar skeletal muscle index (SMI) was calculated by normalizing skeletal muscle area by height $\left(\mathrm{m}^{2}\right)$ and reported as $\mathrm{cm}^{2} / \mathrm{m}^{2}$. For classification of patients as sarcopenic versus non-sarcopenic, a cutoff value was adopted, which was calculated based on a Korean population study consisting of healthy men and women aged 20-39 from the Fourth Korean National Health and Nutritional Examination Surveys (KNHANES IV) conducted in 20082009 [15]. As the KNHANES IV survey used appendicular skeletal muscle mass (ASM), which is measured using dualenergy X-ray absorptiometry to define a cutoff value of sarcopenia, the calculated lumbar SMI $\left(\mathrm{cm}^{2} / \mathrm{m}^{2}\right)$ value was converted into ASM $/$ height ${ }^{2}\left(\mathrm{~kg} / \mathrm{m}^{2}\right)$ using the following formula. 
Appendicular skeletal muscle $/$ height $^{2}\left(\mathrm{~kg} / \mathrm{m}^{2}\right)=0.11 \times$ [skeletal muscle area at $\mathrm{L} 3$ using CT $/$ height $\left.{ }^{2}\left(\mathrm{~cm}^{2} / \mathrm{m}^{2}\right)\right]+1.17$ [16]

Class I sarcopenia was indicated by definition in participants whose height- or weight-adjusted ASM was from 1 to 2 standard deviations (SD) below the mean for young adults, and class II sarcopenia was indicated by definition in participants whose height- or weight-adjusted ASM was below 2 SD [17]. By KNHANES IV survey data, mean ASM/ height ${ }^{2} \pm S D\left(\mathrm{~kg} / \mathrm{m}^{2}\right)$ was $8.42 \pm 0.92$ for men and $6.18 \pm 0.79$ for women. Cutoff value for class I and class II sarcopenia was $7.50 \mathrm{~kg} / \mathrm{m}^{2}$ for men and $5.38 \mathrm{~kg} / \mathrm{m}^{2}$ for women, and 6.58 $\mathrm{kg} / \mathrm{m}^{2}$ for men and $4.59 \mathrm{~kg} / \mathrm{m}^{2}$ for women, respectively [15].

\section{Statistical analyses}

The primary outcomes of interest were OS, defined as the time from the date of initiation of the first-line chemotherapy to the date of death from any cause. Patients who were alive at last follow-up or lost to follow-up were censored at the date of the last follow-up. Patient characteristics were summarized using descriptive methods, and survival analyses were performed by Kaplan-Meier estimate. Continuous clinical and laboratory variables were dichotomized for analytical convenience. The cutoff point for dichotomizing laboratory data was reference values for each variable in our institution. For analysis of associations between sarcopenia and clinicolaboratory factors, Student's t test was used for continuous variables and Fisher exact test was used for categorical variables. Univariate analyses for OS were performed using log-rank test, and subsequent multivariable analysis with Cox proportional hazard model was performed for factors that were significant in univariate analysis. Forward stepwise selection method was used for the prognostic model for OS. If there were missing data, statistical analyses were performed with complete case analyses, in which patients without complete data on the required variables were excluded from particular analyses. The assumption of proportional hazards and linearity for the Cox proportional hazard model was evaluated by log minus log survival plot and by scattered plot between martingale residual and linear predictor score, respectively. Goodness of fit for the final model was assessed by calculating the likelihood ratio test. The reliability of the predictors for prognosis was finally assessed using a bootstrap resampling technique with 1,000 samples $[18,19]$. For each sample, analysis was performed using a stepwise Cox proportional hazard model. If the final stepwise model variables occur in a majority $(>50 \%)$ of the bootstrap models, the original final stepwise regression model can be judged as stable. Statistical analyses were performed using SPSS ver. 18.0 (SPSS Inc., Chicago, IL) and SAS ver. 9.3 (SAS Institute Inc., Cary, NC), and $\mathrm{p}<0.05$ (two-
Table 1. Baseline patient characteristics

\begin{tabular}{|c|c|}
\hline Characteristic & No. $(\%)(\mathrm{n}=88)$ \\
\hline Age, median (range) & $65(34-83)$ \\
\hline \multicolumn{2}{|l|}{ Sex } \\
\hline Male & $59(67.0)$ \\
\hline Female & $29(33.0)$ \\
\hline \multicolumn{2}{|l|}{ Primary pancreatic tumor site } \\
\hline Head & $45(51.1)$ \\
\hline Body & $25(28.4)$ \\
\hline Tail & $18(20.5)$ \\
\hline \multicolumn{2}{|l|}{ Differentiation } \\
\hline Well differentiated & $2(2.3)$ \\
\hline Moderately differentiated & $35(39.8)$ \\
\hline Poorly differentiated & $17(19.3)$ \\
\hline Cytology & $34(38.6)$ \\
\hline \multicolumn{2}{|l|}{ ECOG PS } \\
\hline 0 & $6(6.8)$ \\
\hline 1 & $55(62.5)$ \\
\hline 2 & $27(30.7)$ \\
\hline \multicolumn{2}{|l|}{ Disease status } \\
\hline Recurrent & $24(27.3)$ \\
\hline Initially metastatic & $64(72.7)$ \\
\hline Diabetes mellitus & $26(29.5)$ \\
\hline Weight loss & $20(22.7)$ \\
\hline Biliary drainage & $11(12.5)$ \\
\hline \multicolumn{2}{|l|}{ Previous therapy } \\
\hline Pancreatectomy & $35(39.8)$ \\
\hline Adjuvant therapy & $17(19.3)$ \\
\hline \multicolumn{2}{|l|}{ Sites of metastases } \\
\hline Lymph node & $57(64.8)$ \\
\hline Liver & $53(60.2)$ \\
\hline Peritoneum & $24(27.3)$ \\
\hline Lung & $11(12.5)$ \\
\hline Bone & $10(11.4)$ \\
\hline \multicolumn{2}{|l|}{ No. of metastasized organs } \\
\hline 1 & $28(31.8)$ \\
\hline 2 & $38(43.2)$ \\
\hline 3 & $15(17.0)$ \\
\hline 4 & $7(8.0)$ \\
\hline CA19-9, median (range, U/mL) & $215.00(1.26-62,803.00)$ \\
\hline CEA, median (range, ng/mL) & $3.31(0.11-298.30)$ \\
\hline \multicolumn{2}{|l|}{ First-line chemotherapy } \\
\hline Gemcitabine monotherapy & $29(33.0)$ \\
\hline Gemcitabine+erlotinib & $59(67.0)$ \\
\hline
\end{tabular}

ECOG PS, Eastern Cooperative Oncology Group performance status; CA19-9, carbohydrate antigen 19-9; CEA, carcinoembryonic antigen. 
Table 2. SMI, ASM, and sarcopenia of patients

\begin{tabular}{lrrr} 
Variable & Overall $(\mathbf{n}=\mathbf{8 8})$ & Male $(\mathbf{n = 5 9 )}$ & Female $(\mathbf{n = 2 9 )}$ \\
BMI $\left(\mathbf{k g} / \mathbf{m}^{2}\right)$ & $21.3 \pm 3.0$ & $21.0 \pm 2.7$ & $21.9 \pm 3.3$ \\
SMI $\left(\mathbf{c m}^{2} / \mathbf{m}^{2}\right)$ & $39.5 \pm 7.6$ & $40.8 \pm 7.5$ & $36.8 \pm 6.9$ \\
ASM $\left(\mathbf{k g} / \mathbf{m}^{2}\right)$ & $5.5 \pm 0.8$ & $5.7 \pm 0.8$ & $5.2 \pm 0.8$ \\
Sarcopenia & $76(86.3)$ & $57(96.6)$ & $19(65.5)$ \\
\multicolumn{1}{c}{ Class 1 sarcopenia } & $20(22.7)$ & $7(11.9)$ & $13(44.8)$ \\
Class 2 sarcopenia & $56(63.6)$ & $50(84.7)$ & $6(20.7)$ \\
\hline
\end{tabular}

Values are presented as mean \pm standard deviation or number (\%). SMI, skeletal muscle index; ASM, appendicular skeletal muscle mass; BMI, body mass index.

sided) was considered statistically significant in both univariate and multivariable analysis.

\section{Results}

\section{Patient characteristics}

Eighty-eight patients were included in analysis. Baseline characteristics of the patients are summarized in Table 1. Median age was 65 years (range, 34 to 83 years), and male patients were predominant (59 patients, 67.0\%). Twenty-four patients $(27.3 \%)$ had recurrent disease, while $64(72.7 \%)$ had initially metastatic disease. Lymph node $(64.8 \%)$, liver $(60.2 \%)$, and peritoneum $(27.3 \%)$ were the most common site of metastasis, in order. Gemcitabine alone and gemcitabine plus erlotinib were used for first-line treatment in 29 patients $(33.0 \%)$ and 59 patients $(67.0 \%)$, respectively. Response to treatment in 82 evaluable patients was partial response, stable disease, or progressive disease in four patients $(4.9 \%), 45$ patients $(54.9 \%)$, or 33 patients $(40.2 \%)$.

\section{Sarcopenia and related anthropometric measures}

Data on sarcopenia and related factors are shown in Tables 2 and 3. Seventy-six patients $(86.3 \%)$ had any degree of sarcopenia consisting of class 1 sarcopenia (20 patients, 22.7\%) and class 2 sarcopenia (56 patients, 63.6\%). Age was not associated with sarcopenia, as mean age was 65.5 in sarcopenic patients and 63.3 in non-sarcopenic patients $(\mathrm{p}=0.452)$. However, sarcopenia was more prevalent in men (96.6\% in male vs. $65.5 \%$ in female), particularly class 2 sarcopenia ( $84.7 \%$ vs. $20.7 \%$, p <0.001). As expected, sarcopenia was prevalent in underweight patients. Proportions of sarcopenia patients were $100 \%$ (31 patients out of 31 ) in patients with body mass index (BMI) less than $20 \mathrm{~kg} / \mathrm{m}^{2}, 85.7 \%$
(42 out of 49) with BMI between 20 and $25 \mathrm{~kg} / \mathrm{m}^{2}$, and $37.5 \%$ (3 out of 8 ) with BMI $25 \mathrm{~kg} / \mathrm{m}^{2}$ or higher ( $<<0.001$ ). However, sarcopenia was not associated with recent weight loss $(\mathrm{p}=0.283)$.

\section{OS and univariate analysis for OS}

With a median follow-up period of 44.3 months (range, 0.6 to 44.3 months), median OS for all patients was 5.35 months (95\% confidence interval [CI], 4.11 to 6.59) (Fig. 1). Results of univariate analyses for OS are shown in Table 4. Initially metastatic disease $(p<0.001)$, poor ECOG PS $(p=0.044)$, sarcopenia $(p=0.026)$, liver metastasis $(p=0.015)$, peritoneal metastasis $(p=0.028)$, bone metastasis $(p=0.007)$, anemia $(\mathrm{p}=0.024)$, neutrophilia $(\mathrm{p}<0.001)$, elevated lactate dehydrogenase $(\mathrm{LDH})(\mathrm{p}=0.011)$, hypoalbuminemia $(\mathrm{p}<0.001)$, C-reactive protein elevation ( $p=0.029)$, elevated CEA $(p \leq 0.001)$, and elevated CA19-9 ( $p=0.010)$ were statistically significant.

\section{Multivariable analysis for OS and prognostic model}

Variables with $\mathrm{p}<0.05$ in univariate analysis were selected for entry into the multivariable analysis. In the multivariable analysis (Table 5), elevated CEA ( $p<0.001)$, initially metastatic disease $(p=0.02)$, sarcopenia $(p=0.019)$, neutrophilia ( $p=0.012)$, and elevated LDH ( $p=0.029)$ showed statistical significance and thus were considered independent poor prognostic factors for OS. In internal validation using a bootstrap resampling technique, elevated CEA (bootstrap frequency, $90.6 \%)$, initially metastatic disease $(96.6 \%)$, sarcopenia $(62.7 \%)$, neutrophilia $(78.2 \%)$, and elevated LDH $(78.7 \%)$ were all reliable prognostic factors. For the clinical application, a prognostic score was calculated based on the hazard function derived from the five factors identified in the Cox proportional hazard model. The score was calculated as follows: $0.43 \times(0$, CEA within normal limitation; 1 , CEA elevation $)+0.67 \times(0$, LDH within normal limitation; $1, \mathrm{LDH}$ elevation $)+1.08 \times(0$, absence of neutrophilia; 1 , presence of 
Table 3. Association of clinicolaboratory factors with sarcopenia

\begin{tabular}{|c|c|c|c|}
\hline Variable & Sarcopenia $(\mathrm{n}=76)$ & No sarcopenia $(n=12)$ & p-value \\
\hline Age $(\mathrm{yr})$ & $63.3 \pm 9.6$ & $65.5 \pm 9.1$ & 0.452 \\
\hline \multicolumn{4}{|l|}{ Sex } \\
\hline Male & $57(75.0)$ & $2(16.7)$ & $<0.001$ \\
\hline Female & $19(25.0)$ & $10(83.3)$ & \\
\hline \multicolumn{4}{|l|}{ Disease status } \\
\hline Recurrent & $22(28.9)$ & $2(16.7)$ & 0.5 \\
\hline Initially metastatic & $54(71.1)$ & $10(83.3)$ & \\
\hline \multicolumn{4}{|l|}{ Weight loss } \\
\hline Present & $19(25.0)$ & $1(8.3)$ & 0.283 \\
\hline Absent & $57(75.0)$ & $11(91.7)$ & \\
\hline \multicolumn{4}{|l|}{ BMI $\left(\mathrm{kg} / \mathrm{m}^{2}\right)$} \\
\hline$<20$ & $31(40.8)$ & 0 & $<0.001$ \\
\hline $20-25$ & $42(55.3)$ & $7(58.3)$ & \\
\hline$>25$ & $3(3.9)$ & $5(41.7)$ & \\
\hline \multicolumn{4}{|l|}{ ECOG PS } \\
\hline $0-1$ & $54(71.1)$ & $7(58.3)$ & 0.501 \\
\hline 2 & $22(28.9)$ & $5(41.7)$ & \\
\hline \multicolumn{4}{|l|}{ Neutrophilia } \\
\hline Present & $10(13.3)$ & $1(8.3)$ & 0.704 \\
\hline Absent & $65(86.7)$ & $11(91.7)$ & \\
\hline \multicolumn{4}{|l|}{ Hypoalbuminemia } \\
\hline Present & $10(13.3)$ & 0 & 0.345 \\
\hline Absent & $65(86.7)$ & $12(100)$ & \\
\hline \multicolumn{4}{|l|}{ LDH elevation } \\
\hline Present & $20(28.2)$ & $4(36.4)$ & 0.723 \\
\hline Absent & $51(71.8)$ & $7(63.6)$ & \\
\hline \multicolumn{4}{|l|}{ CA19-9 elevation } \\
\hline Present & $52(70.3)$ & $7(58.3)$ & 0.505 \\
\hline Absent & $22(29.7)$ & $5(41.7)$ & \\
\hline \multicolumn{4}{|l|}{ CEA elevation } \\
\hline Present & $31(44.3)$ & 0 & 0.003 \\
\hline Absent & $39(55.7)$ & $12(100)$ & \\
\hline
\end{tabular}

Values are presented as mean \pm standard deviation or number (\%). BMI, body mass index; ECOG PS, Eastern Cooperative Oncology Group performance status; LDH, lactate dehydrogenase; CA19-9, carbohydrate antigen 19-9; CEA, carcinoembryonic antigen.

neutrophilia $)+1.09 \times(0$, absence of sarcopenia; 1 , presence of sarcopenia $)+1.21 \times(0$, recurrent disease; 1 , initially metastatic disease). The individual prognostic score values for the patients ranged from 0 to 5.48 , with a median value of 2.30 . The patients were then categorized according to three groups based on prognostic scores (33 percentile and 66 percentile): the favorable risk group had a prognostic score $\leq 2.18(\mathrm{n}=25)$, the intermediate risk group had a prognostic score of $>2.18$ but $\leq 3.07(n=26)$, and the poor risk group had a prognostic score $>3.07(n=26)$. The resulting Kaplan-Meier curves for the three groups indicated marked differences in survival $(p<0.001)$ (Fig. 2). Median OS for the favorable, intermedi- ate, and poor risk groups was 11.04 months (95\% CI, 8.14 to 13.93), 5.36 months (95\% CI, 3.02 to 7.70 ), and 2.17 months ( $95 \%$ CI, 0.40 to 3.93), respectively. Six-month OS rates were $88 \%, 50 \%$, and $12 \%$, and 1 -year OS rate was $32 \%, 8 \%$, and $0 \%$, respectively.

\section{Discussion}

In this study, we found five independent prognostic fac- 


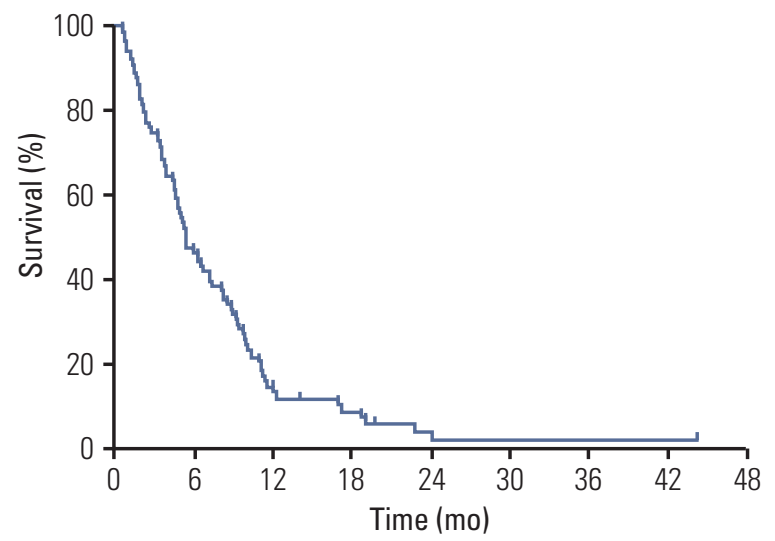

Fig. 1. Kaplan-Meier curve for overall survival. With a median follow-up period of 44.32 months, median overall survival was 5.35 months (95\% confidence interval, 4.11 to 6.59).

tors in patients with recurrent or metastatic pancreatic cancer who received gemcitabine-based chemotherapy: elevated CEA, initially metastatic disease, sarcopenia, neutrophilia, and elevated LDH. In internal validation using a bootstrap resampling technique, all of those factors were reliable prognostic factors. In addition, we proposed a prognostic stratification model using these five factors, which showed a markedly different survival rate for each risk group categorized by prognostic score.

In management of patients with cancer, a well validated prognostic model has an important role in predicting life expectancy, guiding treatment selection, stratifying clinical trial enrollment, analyzing results of clinical studies, and educating patients and their families. Unfortunately, there is no well validated and widely accepted prognostic model for routine application in everyday practice or clinical trial for pancreatic cancer. As published data on prognostic factors for pancreatic cancer consist of heterogeneous status of disease, i.e., curatively resected, recurrent after curative resection, initially metastatic, locally advanced unresectable, or locally advanced borderline resectable, it is difficult to draw a firm conclusion from previous reports. For example, the authors of a literature review found 36 prognostic factor studies reporting a total of 34 possible prognostic factors for advanced pancreatic cancer patients [20].

In the current study, elevated CEA, initially metastatic disease, sarcopenia, neutrophilia, and elevated LDH were statistically significant adverse prognostic factors. These prognostic factors are readily available to treating physicians before chemotherapy, and we expect that their use will aid in clinical decision making and risk stratification.
While CA19-9 is the most widely known tumor marker in pancreatic cancer, a role of CEA in pancreatic cancer is controversial. In the current study, both CA19-9 and CEA were statistically significant prognostic factors in univariate analysis, but only CEA remained significant after multivariable analysis with a Cox proportional hazard model. Some authors reported a prognostic role of CA19-9 for metastatic pancreatic cancer $[21,22]$, but others reported that CEA was more important than CA19-9 [23,24], like our study. Serum level of LDH has been thought to correlate with tumor burden and reflect rapid tumor growth. LDH is a well-established prognostic factor for some cancers including lymphoma and renal cell carcinoma. However, in pancreatic cancer, the role of serum LDH level is poorly defined. To our knowledge, three studies reported serum LDH as an independent prognostic factor for metastatic pancreatic cancer $[20,25,26]$.

Sarcopenia is a syndrome characterized by progressive and generalized loss of skeletal muscle mass and strength with a risk of adverse outcomes including physical disability, poor quality of life, and death [27]. The European Working Group on Sarcopenia in Older People recommends use of normative (healthy young adults) rather than other predictive reference populations, with cutoff points at two SDs below the mean reference value [27]. Because the "normative healthy young adult population" is heterogeneous according to ethnicity, geographic region, or nation, we used a cutoff point deduced from KNHANES IV data [15] instead of the cutoff point for a Western population [11].

Routine measurement of muscle area and calculation of SMI or ASM is inconvenient in a clinical practice setting, and a domestic normative cutoff value might not be available for some regions. However, weight itself is not precise enough for detection of cancer cachexia, as overweight or obese populations often harbor occult, severe pre-existing muscle depletion and prognosis of sarcopenic obesity patients is poorer than that of non-sarcopenic obesity patients [28]. Indeed, in the current study, BMI showed no prognostic significance in univariate analysis $(\mathrm{p}=0.952)$. In addition, recent weight loss, defined as weight loss $>5 \%$ over the past 6 months in the absence of simple starvation [11], is subjective as it is dependent on patients' memory. In our study, sarcopenia did not show a statistically significant association with weight loss ( $p=0.283)$. Therefore, development of a simple and objective tool for measurement of cachexia and sarcopenia is required to assist physicians in adoption of sarcopenia in clinical practice.

The current study has some limitations. First, this analysis is based on retrospective data from small patient population, thus biases inherent to retrospective studies could not be completely avoided. Second, only patients with metastatic or recurrent disease treated with gemcitabine-based chemother- 
Table 4. Log-rank test for overall survival

\begin{tabular}{|c|c|c|c|c|}
\hline \multirow{2}{*}{ Variable } & \multicolumn{2}{|c|}{ No. of patients } & \multirow{2}{*}{ Median OS (95\% CI) } & \multirow{2}{*}{ p-value } \\
\hline & Death & Total & & \\
\hline \multicolumn{5}{|l|}{ Age } \\
\hline Under median & 44 & 47 & $4.90(4.06-5.73)$ & 0.327 \\
\hline Over median & 38 & 40 & $6.54(3.48-9.59)$ & \\
\hline \multicolumn{5}{|l|}{ Sex } \\
\hline Male & 56 & 59 & $5.26(4.44-6.07)$ & 0.104 \\
\hline Female & 26 & 28 & $5.98(3.42-8.54)$ & \\
\hline \multicolumn{5}{|l|}{ Primary site } \\
\hline Head & 42 & 44 & $5.98(3.72-8.24)$ & 0.191 \\
\hline Body & 24 & 25 & $4.90(4.31-5.49)$ & \\
\hline Tail & 16 & 18 & $5.16(3.59-6.73)$ & \\
\hline \multicolumn{5}{|l|}{ Disease status } \\
\hline Recurrent & 21 & 24 & $10.12(7.44-12.80)$ & $<0.001$ \\
\hline Metastatic & 61 & 63 & $4.73(3.52-5.95)$ & \\
\hline \multicolumn{5}{|l|}{ First-line regimen } \\
\hline Gemcitabine & 29 & 29 & $5.98(3.38-8.58)$ & 0.323 \\
\hline Gemcitabine+erlotinib & 53 & 58 & $5.26(4.36-6.16)$ & \\
\hline \multicolumn{5}{|l|}{ ECOG PS } \\
\hline $0-1$ & 57 & 61 & $7.13(4.08-10.18)$ & 0.044 \\
\hline 2 & 25 & 26 & $3.42(1.20-5.63)$ & \\
\hline \multicolumn{5}{|l|}{ Sarcopenia } \\
\hline No & 10 & 11 & $9.27(4.87-13.66)$ & 0.026 \\
\hline Yes & 72 & 76 & $5.16(4.46-5.86)$ & \\
\hline \multicolumn{5}{|l|}{ BMI $\left(\mathrm{kg} / \mathrm{m}^{2}\right)$} \\
\hline$<20$ & 29 & 31 & $5.36(3.96-6.75)$ & 0.952 \\
\hline $20-24.9$ & 46 & 49 & $4.63(3.28-5.98)$ & \\
\hline$\geq 25$ & 8 & 8 & $7.13(3.35-10.91)$ & \\
\hline \multicolumn{5}{|l|}{ LN metastasis } \\
\hline No & 28 & 31 & $6.24(3.99-8.50)$ & 0.113 \\
\hline Yes & 54 & 56 & $4.73(3.69-5.78)$ & \\
\hline \multicolumn{5}{|l|}{ Liver metastasis } \\
\hline No & 31 & 34 & $8.21(5.63-10.80)$ & 0.015 \\
\hline Yes & 51 & 53 & $4.73(3.53-5.93)$ & \\
\hline \multicolumn{5}{|l|}{ Lung metastasis } \\
\hline No & 72 & 76 & $5.36(3.81-6.90)$ & 0.697 \\
\hline Yes & 10 & 11 & $5.36(0.00-11.03)$ & \\
\hline \multicolumn{5}{|l|}{ Peritoneal metastasis } \\
\hline No & 59 & 63 & $6.24(4.20-8.29)$ & 0.028 \\
\hline Yes & 23 & 24 & $4.67(2.65-6.68)$ & \\
\hline \multicolumn{5}{|l|}{ Bone metastasis } \\
\hline No & 73 & 78 & $5.98(4.65-7.31)$ & 0.007 \\
\hline Yes & 9 & 9 & $4.50(1.91-7.10)$ & \\
\hline \multicolumn{5}{|l|}{ Anemia } \\
\hline No & 33 & 37 & $7.33(4.94-9.72)$ & 0.024 \\
\hline Yes & 49 & 49 & $4.73(4.01-5.45)$ & \\
\hline \multicolumn{5}{|l|}{ Neutrophilia } \\
\hline No & 71 & 75 & $6.24(4.80-7.69)$ & $<0.001$ \\
\hline Yes & 11 & 11 & $1.18(0.00-2.67)$ & \\
\hline \multicolumn{5}{|l|}{ Thrombocytosis } \\
\hline No & 70 & 74 & $5.39(3.93-6.84)$ & 0.391 \\
\hline Yes & 12 & 12 & $3.52(0.00-7.48)$ & \\
\hline
\end{tabular}


Table 4. Continued

\begin{tabular}{|c|c|c|c|c|}
\hline \multirow{2}{*}{ Variable } & \multicolumn{2}{|c|}{ No. of patients } & \multirow{2}{*}{ Median OS (95\% CI) } & \multirow{2}{*}{ p-value } \\
\hline & Death & Total & & \\
\hline \multicolumn{5}{|c|}{ LDH elevation } \\
\hline No & 53 & 57 & $6.24(4.70-7.78)$ & 0.011 \\
\hline Yes & 24 & 24 & $3.25(0.57-5.93)$ & \\
\hline \multicolumn{5}{|c|}{ ALT elevation } \\
\hline No & 61 & 64 & $5.98(4.35-7.61)$ & 0.107 \\
\hline Yes & 22 & 23 & $2.73(1.08-4.37)$ & \\
\hline \multicolumn{5}{|c|}{ ALP elevation } \\
\hline No & 53 & 56 & $6.54(4.41-8.67)$ & 0.172 \\
\hline Yes & 30 & 31 & $4.50(2.24-6.76)$ & \\
\hline \multicolumn{5}{|c|}{ Hypoalbuminemia } \\
\hline No & 72 & 76 & $6.18(4.58-7.78)$ & $<0.001$ \\
\hline Yes & 10 & 10 & $2.14(0.86-3.41)$ & \\
\hline \multicolumn{5}{|c|}{ CRP elevation } \\
\hline No & 25 & 27 & $8.18(6.34-10.02)$ & 0.029 \\
\hline Yes & 45 & 45 & $3.91(2.27-5.55)$ & \\
\hline \multicolumn{5}{|c|}{ CEA elevation } \\
\hline No & 48 & 50 & $8.51(6.12-10.90)$ & $<0.001$ \\
\hline Yes & 30 & 31 & $3.42(1.98-4.85)$ & \\
\hline \multicolumn{5}{|c|}{ CA19-9 elevation } \\
\hline No & 23 & 26 & $7.13(2.70-11.56)$ & 0.010 \\
\hline Yes & 58 & 59 & $4.90(3.69-6.10)$ & \\
\hline
\end{tabular}

OS, overall survival; CI, confidence interval; ECOG PS, Eastern Cooperative Oncology Group performance status; BMI, body mass index; LN, lymph node; LDH, lactate dehydrogenase; ALT, alanine transferase; ALP, alkaline phosphatase; CRP, C-reactive protein; CEA, carcinoembryonic antigen; CA19-9, carbohydrate antigen 19-9.

Table 5. Cox-proportional hazard modeling for overall survival

\begin{tabular}{lccrr} 
Variable & Cox model coefficient $(\boldsymbol{\beta})$ & HR & 95\% CI for HR & p-value \\
CEA & 1.430 & 4.18 & $1.95-8.97$ & $<0.001$ \\
Disease status & 1.214 & 3.37 & $1.55-7.32$ & 0.002 \\
Sarcopenia & 1.088 & 2.97 & $1.20-7.36$ & 0.019 \\
Neutrophilia & 1.077 & 2.94 & $1.27-6.79$ & 0.012 \\
LDH elevation & 0.673 & 1.96 & $1.07-3.58$ & 0.029 \\
\hline
\end{tabular}

$\mathrm{HR}$, hazard ratio; $\mathrm{CI}$, confidence interval; CEA, carcinoembryonic antigen; LDH, lactate dehydrogenase.

apy were included. Although uniform inclusion criteria might make this analysis more homogeneous, we could not confirm the prognostic role of the current prognostic model in patients with locally advanced pancreatic cancer or patients who underwent other therapies such as fluorouracil, leucovorin, irinotecan, and oxaliplatin (FOLFIRINOX) or nanoalbumin paclitaxel. A methodologically robust, wellpowered prognostic model for advanced pancreatic cancer should be developed in the future.

\section{Conclusion}

In summary, five independent prognostic factors in patients with recurrent or metastatic pancreatic cancer who received gemcitabine-based chemotherapy were identified, and a prognostic model consisting of these factors was proposed. This prognostic model can be helpful in prediction of prognosis in clinical practice and can be used as a stratifica- 


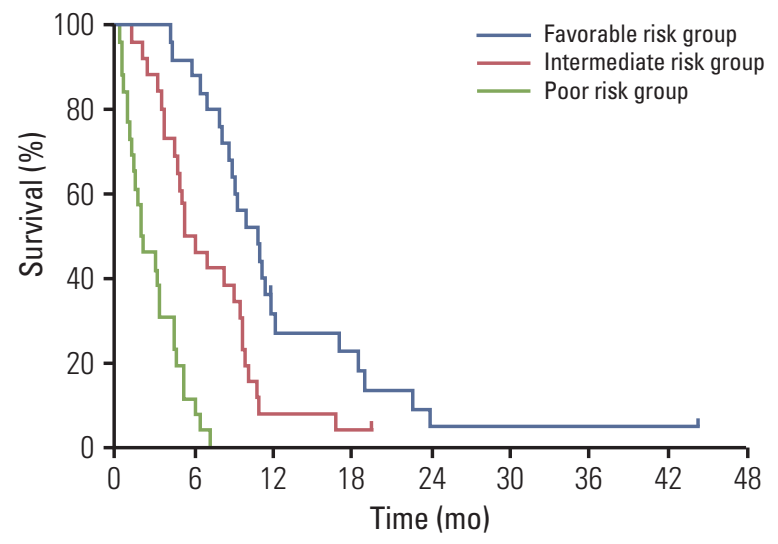

Fig. 2. Kaplan-Meier curve for overall survival according to prognostic grouping. Median overall survival for the favorable (blue line), intermediate (red line), and poor risk groups (green line) was 11.04 months (95\% confidence interval [CI], 8.14 to 13.93), 5.36 months (95\% CI, 3.02 to 7.70), and 2.17 months (95\% CI, 0.40 to 3.93 ), respectively $(\mathrm{p}<0.001)$. tion factor for clinical trials, if confirmed in future validation in a larger population.

\section{Conflicts of Interest}

Conflict of interest relevant to this article was not reported.

\section{Acknowledgments}

This work was supported by the National Research Foundation of Korea (NRF) grant funded by the Korea government (MSIP) (No. 2015R1C1A1A01054452).

\section{References}

1. Torre LA, Bray F, Siegel RL, Ferlay J, Lortet-Tieulent J, Jemal A. Global cancer statistics, 2012. CA Cancer J Clin. 2015;65: 87-108.

2. Hidalgo M. Pancreatic cancer. N Engl J Med. 2010;362: 1605-17.

3. Burris HA 3rd, Moore MJ, Andersen J, Green MR, Rothenberg ML, Modiano MR, et al. Improvements in survival and clinical benefit with gemcitabine as first-line therapy for patients with advanced pancreas cancer: a randomized trial. J Clin Oncol. 1997;15:2403-13.

4. Moore MJ, Goldstein D, Hamm J, Figer A, Hecht JR, Gallinger $\mathrm{S}$, et al. Erlotinib plus gemcitabine compared with gemcitabine alone in patients with advanced pancreatic cancer: a phase III trial of the National Cancer Institute of Canada Clinical Trials Group. J Clin Oncol. 2007;25:1960-6.

5. Conroy T, Desseigne F, Ychou M, Bouche O, Guimbaud R, Becouarn $Y$, et al. FOLFIRINOX versus gemcitabine for metastatic pancreatic cancer. N Engl J Med. 2011;364:1817-25.

6. Von Hoff DD, Ervin T, Arena FP, Chiorean EG, Infante J, Moore $\mathrm{M}$, et al. Increased survival in pancreatic cancer with nab-paclitaxel plus gemcitabine. N Engl J Med. 2013;369: 1691-703.

7. Jung KW, Won YJ, Kong HJ, Oh CM, Cho H, Lee DH, et al. Cancer statistics in Korea: incidence, mortality, survival, and prevalence in 2012. Cancer Res Treat. 2015;47:127-41.

8. Cartwright TH, Ginsburg A, Wilfong LS, Harrell RK, Hoverman JR. Use of first-line chemotherapy for advanced pancreatic cancer: FOLFIRINOX versus gemcitabine-based therapy.
J Clin Oncol. 2014;32(5S):Abstr 4132.

9. Bachmann J, Heiligensetzer M, Krakowski-Roosen H, Buchler MW, Friess H, Martignoni ME. Cachexia worsens prognosis in patients with resectable pancreatic cancer. J Gastrointest Surg. 2008;12:1193-201.

10. Davison KK, Birch LL. Lean and weight stable: behavioral predictors and psychological correlates. Obes Res. 2004;12: 1085-93.

11. Fearon K, Strasser F, Anker SD, Bosaeus I, Bruera E, Fainsinger $\mathrm{RL}$, et al. Definition and classification of cancer cachexia: an international consensus. Lancet Oncol. 2011;12:489-95.

12. Dalal S, Hui D, Bidaut L, Lem K, Del Fabbro E, Crane C, et al. Relationships among body mass index, longitudinal body composition alterations, and survival in patients with locally advanced pancreatic cancer receiving chemoradiation: a pilot study. J Pain Symptom Manage. 2012;44:181-91.

13. Di Sebastiano KM, Yang L, Zbuk K, Wong RK, Chow T, Koff $\mathrm{D}$, et al. Accelerated muscle and adipose tissue loss may predict survival in pancreatic cancer patients: the relationship with diabetes and anaemia. Br J Nutr. 2013;109:302-12.

14. Tan BH, Birdsell LA, Martin L, Baracos VE, Fearon KC. Sarcopenia in an overweight or obese patient is an adverse prognostic factor in pancreatic cancer. Clin Cancer Res. 2009;15: 6973-9.

15. Kim YS, Lee Y, Chung YS, Lee DJ, Joo NS, Hong D, et al. Prevalence of sarcopenia and sarcopenic obesity in the Korean population based on the Fourth Korean National Health and Nutritional Examination Surveys. J Gerontol A Biol Sci Med 
Sci. 2012;67:1107-13.

16. Mourtzakis M, Prado CM, Lieffers JR, Reiman T, McCargar LJ, Baracos VE. A practical and precise approach to quantification of body composition in cancer patients using computed tomography images acquired during routine care. Appl Physiol Nutr Metab. 2008;33:997-1006.

17. Janssen I, Heymsfield SB, Ross R. Low relative skeletal muscle mass (sarcopenia) in older persons is associated with functional impairment and physical disability. J Am Geriatr Soc. 2002;50:889-96.

18. Brunelli A, Rocco G. Internal validation of risk models in lung resection surgery: bootstrap versus training-and-test sampling. J Thorac Cardiovasc Surg. 2006;131:1243-7.

19. Grunkemeier GL, Wu Y. Bootstrap resampling methods: something for nothing? Ann Thorac Surg. 2004;77:1142-4.

20. Stocken DD, Hassan AB, Altman DG, Billingham LJ, Bramhall SR, Johnson PJ, et al. Modelling prognostic factors in advanced pancreatic cancer. Br J Cancer. 2008;99:883-93.

21. Marechal R, Demols A, Gay F, De Maertelaere V, Arvanitaki M, Hendlisz A, et al. Prognostic factors and prognostic index for chemonaive and gemcitabine-refractory patients with advanced pancreatic cancer. Oncology. 2007;73:41-51.

22. Wasan HS, Springett GM, Chodkiewicz C, Wong R, Maurel J, Barone C, et al. CA 19-9 as a biomarker in advanced pancreatic cancer patients randomised to gemcitabine plus axitinib or gemcitabine alone. Br J Cancer. 2009;101:1162-7.
23. Lee KJ, Yi SW, Chung MJ, Park SW, Song SY, Chung JB, et al. Serum CA 19-9 and CEA levels as a prognostic factor in pancreatic adenocarcinoma. Yonsei Med J. 2013;54:643-9.

24. Inal A, Kos FT, Algin E, Yildiz R, Berk V, Tugba Unek I, et al. Prognostic factors in patients with advanced pancreatic cancer treated with gemcitabine alone or gemcitabine plus cisplatin: retrospective analysis of a multicenter study. J BUON. 2012; 17:102-5.

25. Tas F, Aykan F, Alici S, Kaytan E, Aydiner A, Topuz E. Prognostic factors in pancreatic carcinoma: serum LDH levels predict survival in metastatic disease. Am J Clin Oncol. 2001;24: 547-50.

26. Faloppi L, Bianconi M, Giampieri R, Sobrero A, Labianca R, Ferrari $\mathrm{D}$, et al. The value of lactate dehydrogenase serum levels as a prognostic and predictive factor for advanced pancreatic cancer patients receiving sorafenib. Oncotarget. 2015;6: 35087-94.

27. Cruz-Jentoft AJ, Baeyens JP, Bauer JM, Boirie Y, Cederholm T, Landi F, et al. Sarcopenia: European consensus on definition and diagnosis: report of the European Working Group on Sarcopenia in Older People. Age Ageing. 2010;39:412-23.

28. Martin L, Birdsell L, Macdonald N, Reiman T, Clandinin MT, McCargar LJ, et al. Cancer cachexia in the age of obesity: skeletal muscle depletion is a powerful prognostic factor, independent of body mass index. J Clin Oncol. 2013;31:1539-47. 